\title{
USOS DE LA PARTÍCULA COMO EN APRENDIENTES FRANCÓFONOS, ALEMANES Y ANGLOSAJONES EN ESPAÑOL COMO LENGUA EXTRANJERA'
}

\author{
USOS DA PARTÍCULA COMO EM APRENDIZES FRANCÓFONOS, \\ ALEMÃES E ANGLO-SAXÕES EM ESPANHOL \\ COMO LÍNGUA ESTRANGEIRA
}

\author{
Anita Ferreira* \\ Lorena Blanco* \\ Jessica Elejalde ${ }^{* * *}$
}

\begin{abstract}
RESUMEN
La problemática que se aborda en el presente artículo se relaciona con las dificultades que se observan en el uso de la partícula como en aprendientes de ELE de nivel de competencia A2 y B1 con L1 francés, alemán e inglés. Para este fin, se realiza un estudio de Corpus de Aprendientes en formato digital (del inglés, Computer Learner Corpora, CLC). Las variables corresponden a la tendencia de uso de la partícula como según el nivel de competencia A2 y B1 y la lengua materna. A partir del corpus CAELE se ha constituido un conjunto de 1578 enunciados textuales digitales de aprendientes anglosajones, francófonos y alemanes de ELE. Los objetivos principales son (1) determinar los usos más frecuentes de la partícula como de acuerdo con los usos atributivo de ejemplificación, relativo modal y comparativo y (2) delimitar los usos más frecuentes de acuerdo con el nivel de competencia y lengua materna de los aprendientes en estudio". Los resultados muestran un mayor uso de la partícula como en función de atributo ejemplificativo, seguido por el uso de relativo de modo y el comparativo de igualdad. En cuanto a la L1 y nivel de competencia los aprendientes franc y nivel de competencia ucturas en estudio e modo y comparativo de igualdad.arativo, mans se observa la mayor frecuencia de uso ófonos de B1 evidencian un mayor número de usos en las estructuras en estudio, seguidos de los alemanes de nivel A2 y de los anglosajones de nivel B1.

Palabras clave: corpus de aprendientes en formato computacional; partícula como; el uso del como en ELE; Español para fines académicos.
\end{abstract}

\section{RESUMO}

O problema abordado neste artigo está relacionado às dificuldades observadas no uso da partícula como em aprendizes do ELE dos níveis de proficiência A2 e B1 com francês, alemão e inglês L1. Para tal, é realizado um estudo do Corpus of Learners em formato digital (Computer Learner Corporation, CLC). As variáveis correspondem à tendência de uso da partícula de acordo com o nível de competência A2 e B1 e a língua materna. A partir do corpus CAELE, foi constituído um conjunto de 1578 sentenças textuais digitais de aprendizes anglo-saxões, francófonos e alemães de ELE. Os objetivos principais são (1) determinar os usos mais frequentes da partícula como de acordo com os usos atributivos de exemplificação, modal relativo e comparativo e (2) delimitar os usos mais frequentes de acordo com o nível de competência e língua materna do aprendizes em estudo". Os resultados mostram maior uso da partícula em função de atributo exemplar, seguido do uso de modo relativo e igualdade comparativa. Em relação ao L1 e ao nível de competência, os aprendizes francófonos de B1 apresentam um maior número de utilizações nas estruturas em estudo, seguidos dos alemães do nível A2 e dos anglo-saxões do nível B1.

Palavras-chave: corpus de alunos em formato computacional, partícula como, o uso de como em ELE, espanhol para fins acadêmicos.

\section{INTRODUCCIÓN}

Al consultar la entrada léxica como, en el Diccionario de la RAE (2014), esta se define dieciocho veces. Algunas acepciones incluyen la definición del como en calidad de estructura caracterizada por el desuso, por tanto, sus usos se pueden reducir a catorce. Destacan las funciones de adverbio relativo y de como comparativo. Además, se precisa que esta palabra esencialmente funciona como adverbio y conector. También en algunos contextos oracionales

* Universidad de Concepción, Concepción, Chile. aferreir@udec.cl

Orcid: https://orcid.org/0000-0001-7979-6467

**Universidad Católica de la Santísima Concepción, Concepción, Chile. lblanco@ucsc.cl Orcid: https://orcid.org/0000-0002-9584-899X

***Universidad Católica de Temuco, Temuco, Chile. jelejalde@uct.cl Orcid: https://orcid.org/0000-0002-6024-2381

1. Este artículo ha sido desarrollado en el contexto del Proyecto Fondecyt 1180974 Diseño e implementación de un corpus escrito de aprendientes de Español como Lengua Extranjera (ELE) para el análisis de la interlengua, 2018-2021. La investigadora responsable es la Dra. Anita Ferreira Cabrera 
puede funcionar como preposición. En el Diccionario Panbispánico de Dudas, la RAE (2005, pág. 150) se refiere a esta partícula como "palabra átona que, como tal, debe escribirse sin tilde, a diferencia del adverbio interrogativo o exclamativo cómo. Puede funcionar como adverbio, como conjunción y como preposición" En este artículo, la problemática que se aborda se relaciona con las dificultades que se observan en los usos de la partícula como en aprendientes de ELE de nivel de competencia A2 y B1 con L1 francés, alemán e inglés. El estudio es descriptivo de carácter mixto, cuantitativo y cualitativo, para delimitar los tipos de usos del como más frecuentes en aprendientes de Español como Lengua Extranjera (ELE) francófonos, alemanes y anglosajones de niveles de competencia A2 y B1.

Diversos autores se han referido a la complejidad y dificultad implicada en tratar de analizar y resolver la funcionalidad de esta partícula en el uso del español como lengua materna (L1). Esta tarea se hace aún más compleja cuando se intenta abordar desde una perspectiva del Español como lengua segunda (L2) o extranjera (LE). González García (1995) señala que el como es una de los elementos de mayor uso funcional como se evidencia en sus variedades de usos como adverbio relativo, conjunción causal, condicional o comparativa (pág. 315). Por otra parte, Trujillo (1990) sostiene que esta forma constituye una sola unidad semántica con distintas distribuciones y diferentes sentidos contextuales, haciendo depender la realidad lingüística de unos parámetros de clasificación externos, tanto desde el punto de vista teórico como metodológico. Para dicho autor el uso del como no pierde en ningún estado o contexto de uso su significado modal relativo. Asimismo, Morera señala que en sí la forma como no significa ni comparación, ni modalidad, ni aproximación, ni condición, etc., sino una especie de correspondencia con la estructura 'del modo que'. Todos los demás usos y significados dependen del contexto y, en este sentido, no siempre pueden clasificarse de modo homogéneo (MORERA, 1990).

Desde un punto de vista metodológico, las investigaciones basadas en corpus de aprendientes han aportado en las descripciones de la interlengua en materia de qué aspectos lingüísticos se aprenden antes que otros, cuáles son más difíciles de aprender y las diferencias entre aprendientes de lenguas maternas distintas. Los corpus de aprendientes como herramientas de investigación posibilitan el desarrollo de nuevos tipos de investigaciones tales como los análisis frecuencias de coaparición o de patrones de uso lingüístico por parte de grupos de aprendientes (GRANGER, 2015; HASSELGARD Y JOHANSSON, 2011; SÁNCHEZ, 2015; FERREIRA Y ELEJALDE, 2020; FERREIRA, BLANCO Y ELEJALDE, 2020). Para encauzar la investigación en este ámbito interdisciplinario, hemos estado implementado un corpus de aprendientes de ELE que hemos denominado CAELE. Este corpus digital permite observar distintas problemáticas lingüísticas y realizar análisis léxico-gramatical, que puede incluir listas de frecuencias, colocaciones, expresiones multipalabras, palabras clave, n-gramas, entre otros.

El artículo se organiza en las siguientes secciones: En la sección 2, nos referimos a los fundamentos teóricos en materia de la partícula como. En la sección 3, abordamos los procedimientos metodológicos del estudio descriptivo sobre la partícula como. En la sección 4, presentamos los resultados cuantitativos y cualitativos. Finalmente, presentamos algunas conclusiones sobre los avances y logros obtenidos en esta investigación.

\section{FUNDAMENTOS TEÓRICOS}

\subsection{Dificultades en los usos de la partícula como en Español}

Como se mencionó en la introducción, varios autores desde distintas perspectivas han intentado delimitar los distintos usos y funciones de la partícula como. A continuación, se especificarán los principales aportes en relación con investigaciones previas. Jiménez (2003, afirma que es "una de las partículas más versátiles del español, pudiéndose encontrar con valores gramaticales de hasta cinco tipos bien diferenciados", de manera que puede considerarse como "una de las más bifurcadas y variadas de entre las palabras estructurales del español, y en ese sentido supera probablemente a la igualmente diversificada que" (pág. 117-123).

Desde una perspectiva semántica, Trujillo (1990) señala que usualmente en los estudios sobre el como no se describe el significado y las posibilidades semánticas del adverbio. En cambio, si abordan los diversos tipos sintácticos del adverbio acorde con el valor contextual en que son usados. Así, por ejemplo, se puede identificar un como modal, un como causal, un como comparativo, entre otros. Así también otras funciones distintas del como son los cómo interrogativos o exclamativos. Trujillo (1990) postula una sola unidad semántica con diferentes distribuciones y variados sentidos textuales, en la cual es importante el punto de vista del aprendiente, es decir, su intuición lingüística (L1) y cómo dicho aprendiente capta o analiza lo real. Con dicho criterio gramatical consistente en 
explicar las construcciones a partir de su estructura semántica idiomática, la partícula como mantiene su significado de modal relativo. Esta idea es similar a lo planteado por Morera (1990) al señalar que «no significa ni comparación, ni modalidad, ni aproximación, ni condición, etc., sino algo así como 'del modo que'. Todo lo demás depende del contexto y, como tales sentidos contextuales, no siempre pueden clasificarse de modo homogéneo» (pág. 691).

En cambio, para Cano Aguilar (1995) el como es la partícula española que con mayor facilidad puede introducir segmentos oracionales y no oracionales; puede funcionar sola, o formando parte de varias correlaciones, o bien apoyándose en otros elementos de la oración en la que se integra. En consonancia con ello, los valores significativos de las construcciones que como constituye son también extraordinariamente diversos.

Acín Villa (2001) hace hincapié en su polivalencia, lo que explicaría, "por una parte, que en gramáticas y estudios de carácter general aparece tratado en secciones muy diversas, recibiendo sólo tratamientos parciales, y, a veces, hasta contradictorios. Y, por otra parte, sin duda a causa de la complejidad que como presenta, que no se le haya prestado mucha atención en estudios monográficos hasta hace unos pocos años".

El adverbio relativo de modo como constituye una unidad idiomática en cuyo significado originario básico se encuentran contenidos los valores de preposición + (artículo) + noción de 'modo' + relativo general. En su actualización en el discurso, condicionado por ciertos factores que concurren en el contexto, manifiesta en diferente grado o reemplaza, adquiriendo el segmento del que forma parte diversos sentidos que sirven de base a la mayor parte de los tratados de gramática para hablar de otros tantos tipos de esta forma (HERNANDO, 2002).

\subsection{La enseñanza de la partícula como según el Plan Curricular del Instituto de Cervantes}

De acuerdo con lo planteado en el Plan Curricular del Instituto Cervantes (2006), el nivel de competencia comunicativa A2 se caracteriza por la enseñanza y la producción del cuantificador propio como, es decir, se establece comparación de igualdad entre dos elementos. Por ejemplo, "Juan es tan alto como yo" o "Juan corre tanto como yo". En este sentido, se puede encontrar el como en oraciones subordinadas adverbiales de tipo comparativas introducidas por: a) tan. Se comparan cualidades de personas, objetos o situaciones. Por ejemplo, "soy tan alto como tú", b) el adjetivo indefinido tanto(a)(s). "Tengo tantos problemas como tú", c) tanto... como, para establecer comparación de frecuencia o de intensidad sobre las acciones. Un ejemplo es: "corre tanto como, yo". Esta proposición equivale a decir es tan rápido como yo o corre con la misma frecuencia que yo. Una característica concreta de este tipo de construcciones es la posición inicial o antepuesta que adopta, vale decir, "corro tanto como tú" es gramatical, mientras que decir "tanto corro como tú *" es una oración agramatical.

En el nivel de competencia B1, la partícula como también aparece en oraciones subordinadas adverbiales causales en posición inicial: "como no venías empecé a cenar". En lo referido a los contenidos gramaticales que se desarrollan en el nivel B1 y B2 según el Marco Común Europeo de Referencia para la Enseñanza de las lenguas (CONSEJO DE EUROPA, 2001), las oraciones relativas, incluidas las introducidas por el adverbio como, se enmarcan en un nivel B1 cuando el antecedente es conocido y en un nivel B2 cuando es desconocido (DÍAZ RODRÍGUEZ, MARTÍNEZ SÁNCHEZ y REDÓ, 1964).

Sobre la base de estos planteamientos, se puede señalar que la producción del como comienza en el nivel inicial con una serie de estructuras, en las cuales las más compleja es la subordinación, por lo que se proyecta al siguiente nivel. En consecuencia, en la enseñanza del ELE se deberían tratar los diferentes usos del adverbio como y las diferentes estructuras sintácticas implicadas, de tal forma que los aprendientes puedan reconocer cuáles son los distintos significados y estructuras del uso del como en la lengua española.

\subsection{Usos del como en estudio}

\subsubsection{La estructura de atributo ejemplificativa introducida por como}

El uso de la partícula como en términos ejemplificativo pone de manifiesto el valor atributivo que se reconoce en esta palabra cuando introduce algún elemento ilustrativo de la noción que se predica. Por ejemplo, personas inteligentes como tú; capitales como Roma o París. En este aspecto, el valor atributivo significa que la expresión del uso como desempeña la función sintáctica del atributo. En palabras de Cuadrado (2002), el uso ejemplificativo, refiere principalmente a la introducción de sintagmas nominales concordados que cumplen los requisitos necesarios para ser interpretados 
como atributos del sujeto, del implemento o suplemento. Funciona también como preposición y, con otros tipos de sintagmas o grupos sintagmáticos, adquiere un valor cuasi-prefijal de atenuación con diferentes matices.

En las estructuras oracionales entró como profesora y, ellos postularon como organizadores del concurso, se observa que estos sintagmas nominales introducidos por como cumplen las condiciones de atribución de características. Es decir, desempeñan la función de atributo del sujeto, del implemento y del suplemento. En estos tipos de construcciones, el uso de como tiene un valor prepositivo, el cual puede ser sustituido por la preposición de o por una locución prepositiva como en concepto de o en calidad de. Así, Cuadrado (2002) explica que:

\begin{abstract}
"Introduciendo grupos sintagmáticos nominales del tipo de Había como quinientas personas, constituidos por la combinación de un determinante numeral cardinal y un núcleo sustantivo, que no desempeñan la función de atributo en ninguna de las variantes apuntadas, tiene un valor intermedio entre el prepositivo, al poder ser sustituido por la locución prepositiva alrededor de, y el cuasi prefijal que se presenta en otras construcciones, principalmente ante un sustantivo (Sentía como remordimiento), adjetivo (Estaba como melancólico), grupo sintagmático nominal introducido por una preposición (Como a la mitad del camino, bay un cruce), oración subordinada con el verbo en gerundio (Tendía los brazos como queriendo abrazarlo) o con el verbo en forma personal introducida por el que: Sentía como que alguien estuviera controlando todos sus movimientos" (pág. 336).
\end{abstract}

La estructura de atributo ejemplificativa presenta esta característica de tener un valor prepositivo, que dificulta la comprensión de esta partícula tanto en hablantes nativos como aprendientes de ELE.

\title{
1.4 El como comparativo
}

En el Diccionario Panbispánico de Dudas, la RAE (2005) define la forma como en cuanto a forma comparativa utilizando dos acepciones: primero, como adjetivo comparativo ante sustantivos. Indica que la cantidad, magnitud o intensidad de lo designado es semejante a otra mencionada o consabida, en correlación con como o, menos a menudo, cuanto. "Hay tanta gente aquí como en la playa". "Hizo tanto ruido como una bomba". Segundo, se usa como cuantificador comparativo en las construcciones comparativas de igualdad. El segundo término va introducido por como. "En nuestro cine, hay casi tantos curas como prostitutas". "Esa noche se esfumó tan sigilosamente como había llegado".

Por su parte, el Manual de la Nueva Gramática de la RAE (2009), define las construcciones comparativas, como: aquellas que establecen una comparación entre dos valores de carácter cuantificativo. Se comparan magnitudes número, cantidad y grado - mediante recursos gramaticales. Se clasifican las construcciones comparativas en función de los cuantificadores comparativos que les dan sentido, por lo tanto, se distinguen las construcciones comparativas en 2 tipos: Las comparativas de desigualdad y las de igualdad.

\subsection{El como adverbio relativo de modo}

En el Diccionario Panbispánico de Dudas, se precisa que el como adverbio relativo de modo, puede llevar o no antecedente explícito; el antecedente puede ser un sustantivo (modo, manera, forma, etc.) o un adverbio (tal, así): "La manera como canta Juan no me gusta", "Lo bice tal como me dijiste", "Se queda así como está"; "Pinté la casa como tú querías". Se hace tónico, aunque sigue escribiéndose sin tilde, cuando se coordina con otro adverbio relativo y no es el último elemento de la coordinación: Me vestiré como y cuando yo quiera. (RAE, 2005).

Brucart (1999) indica que, en general, en las gramáticas el como está caracterizado como un adverbio relativo de naturaleza modal. De tal modo, que su función fundamental es la de introducir cláusulas que expresan una circunstancia modal de la oración principal. Martínez García (1989) explica el comportamiento funcional de los adverbios relativos como, cuando y donde, señalando que si tienen antecedente, convierten a la oración en adjetivo (es decir, la adjetivan); si no llevan antecedente, capacitan a la oración para funcionar como sustantivo merced al valor de artículo que conllevan.

\subsection{Procedimientos Metodológicos}

El diseño del estudio es de tipo descriptivo con un enfoque de análisis de datos mixto: cuantitativo y cualitativo. Los datos de corte cuantitativo permiten identificar las frecuencias y sistematicidad en los errores, así como también determinar las tendencias de uso de la partícula como. Con respecto a los datos cualitativos, estos proporcionarán explicaciones sobre las tendencias de uso encontradas. Metodológicamente la investigación se circunscribe en los procedimientos de la lingüística de corpus. Los procedimientos metodológicos se sustentan en los lineamientos 
propuestos en el Proyecto Fondecyt No.1180974 (FERREIRA, 2018). Dos son los objetivos principales concebidos en el contexto de este artículo:

(1) Determinar los usos más frecuentes de la partícula como de acuerdo con sus especificaciones gramaticales (modal, comparativo y ejemplificativo, etc) en un corpus de aprendientes de ELE

(2) Explicar los usos más frecuentes de acuerdo con el nivel de competencia y lengua materna de los aprendientes de ELE en estudio.

\subsection{El Corpus de Aprendientes de Español como Lengua Extranjera, CAELE}

El Corpus de Aprendientes de ELE es una colección de 1217 textos producidos en un ambiente presencial a través de un aula virtual, procesados a través del software Sketcb Engine (KILGARRIFF; BAISA; BUŠTA; JAKUBÍČEK; KOVÁ̌̌; MICHELFEIT; RYCHLÝ; SUCHOMEL 2014; KILGARRIFF; RYCHLÝ; SMRŽ; TUGWELL, 2004). Estos textos han sido recolectados durante los periodos de clases entre los años 2014 y 2019 y se distribuyen en los niveles de competencia del ELE, A2 y B1. CAELE está conformado por 265.362 palabras, por 296.136 tokens y por 14.902 oraciones. La recolección de los textos se realizó durante los cursos de ELE ofrecidos semestralmente cada año en una universidad chilena. La extensión es de 150 a 200 palabras (A2) y de 250 a 300 (B1). Las temáticas de escritura se determinaron mediante un análisis de necesidades y consideraron temas del ámbito de español para fines académicos científicos y humanísticos: literatura, ciencia y tecnología, cultura latinoamericana, etc. Las secuencias textuales recolectadas en los textos responden a la estructura narrativa, descriptiva y argumentativa (FERREIRA y ELEJALDE, 2019). Para ratificar los niveles de competencia que los aprendientes declaran en una encuesta de registro, se aplicó la prueba de multinivel de competencia (FERREIRA, 2016). Los aprendientes que escribieron los textos son 210 sujetos y provienen de los programas de intercambio de una universidad chilena en el marco de la movilidad estudiantil.

\subsection{Implementación del corpus}

Para el estudio se seleccionaron los textos producidos por aprendientes francófonos, alemanes y anglosajones puesto que son los mayor número en el corpus CAELE. Para el análisis de los textos se realizó una búsqueda de concordancias de los usos de la partícula como con la herramienta Word Sketch de Sketch Engine. Luego, se seleccionaron los usos con mayor frecuencia para filtrar los resultados de concordancias según la L1 de los aprendientes (francés, alemán e inglés) y nivel de competencia A2 y B1. El corpus quedó constituido por un total de 1295 enunciados textuales con todos los usos de la partícula como producidos por los aprendientes francófonos, alemanes y anglófonos de los niveles de competencia A2 y B1.

En la Figura 1 se muestra el tipo de resultados que arroja otra herramienta utilizada de Sketch Engine, la Concordancia. Se puede ver el uso de como en el contexto del enunciado. Además, se ilustra la interfaz con la que funciona el análisis del software Sketch Engine. Los enunciados pueden analizarse de acuerdo con su concordancia, los tipos de palabras, el contexto de la oración y el número de frecuencias o apariciones en los textos.

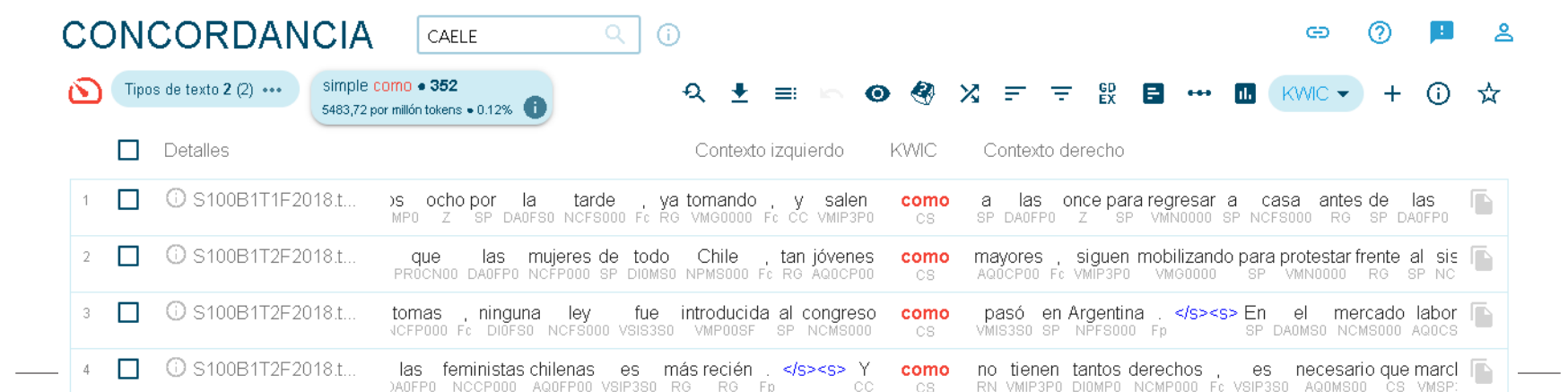

Figura 1. Ejemplos de concordancias en la partícula como en Sketch Engine 


\subsection{La Anotación}

Respecto del análisis y clasificación de los errores se consideró la taxonomía propuesta para el etiquetado de errores con criterio etiológico de Ferreira y Elejalde (2020). Para la identificación de los diferentes usos de la partícula como se consideraron las etiquetas como más el uso específico que se consulta: como-modal; como-ejemplo; comocomparar.

A partir de las acepciones del como registradas en el Diccionario de la RAE (2014), se ha considerado los siguientes usos siguiendo los indicadores por nivel de competencia A2 y B1 según el Plan Curricular de Cervantes (INSTITUTO CERVANTES, 2006).

Tabla 1. Diferentes usos de la partícula como basada en la RAE (2014) (elaboración propia)

\begin{tabular}{|l|l|l|}
\hline Forma & Función & Ejemplo \\
\hline \multirow{4}{*}{ Como } & Adverbio relativo de lugar & $\begin{array}{l}\text { He viajado a distintos lugares como } \\
\text { Talcahuano y Dichato. }\end{array}$ \\
\cline { 2 - 3 } & Adverbio ejemplificativo & $\begin{array}{l}\text { Son varios colores como amarillo, rojo y } \\
\text { verde. }\end{array}$ \\
\cline { 2 - 3 } & Adverbio relativo de tiempo & $\begin{array}{l}\text { No tiene ningún apoyo como durante la } \\
\text { Guerra Fría }\end{array}$ \\
\cline { 2 - 3 } & Adverbio de modo & Me encantaba la manera como sonreía. \\
\cline { 2 - 3 } & $\begin{array}{l}\text { Conector comparativo de igualdad } \\
\text { (como, tan-como, tanto- como) }\end{array}$ & $\begin{array}{l}\text { Es alto como su madre. } \\
\text { Es tan inteligente como su hermana. } \\
\text { Ella tenía tantas ganas de viajar como él. }\end{array}$ \\
\cline { 2 - 3 } & Conector causal & Como no llegaste, tuve que salir. \\
\cline { 2 - 3 } & Adverbio interrogativo indirecto & No sé cómo agradecerte tantos favores. \\
\hline
\end{tabular}

\subsection{Procesamiento de los datos}

Los procedimientos utilizados en el software Sketch Engine para este estudio fueron dos: 1) el uso de Word Scketch, cuyo uso permite identificar el uso de una palabra y que otros modificadores pueden complementar o funcionar con una categoría en especial de la palabra buscada y 2) las concordancias para identificar los contextos de aparición más frecuentes de la partícula como en el corpus CAELE. Respecto a la distribución de los segmentos textuales para el análisis de los usos de la partícula como se cauteló la variable investigador, por lo que se asignaron aleatoriamente para ser revisados por el equipo de investigación.

\section{RESULTADOS Y DISCUSIÓN}

En lo referido al Objetivo 1 "determinar los usos más frecuentes de la partícula como de acuerdo con sus especificaciones gramaticales (modal, comparativo y atributivo ejemplificativo, etc.) en un corpus de aprendientes de ELE" se realizó un análisis de concordancias para delimitar los usos del adverbio como más utilizados en el CAELE en las tres lenguas maternas más representativas del corpus francés, alemán e inglés.

\subsection{Usos del adverbio como más utilizados en el corpus digital CAELE}

En la Tabla 2 se muestra la frecuencia de los usos más frecuentes de la partícula como en el CAELE. Se puede percibir 1246 apariciones del como, las que se distribuyen en 7 usos: ejemplificativo, relativo de modo, comparativo de igualdad, relativo de lugar, conector causal, interrogativo indirecto y relativo de tiempo. Destaca la función de adverbio con valor ejemplificativo (47\%), prácticamente la mitad de los usos corresponden a esta categoría. Asimismo, usos menores contabilizados en el análisis corresponden a casos de adverbios interrogativos en oraciones indirectas (2\%) y a adverbios relativos que expresan una función temporal (1\%). 
Tabla 2. Frecuencias de usos de la partícula como y su porcentaje relativo respecto del total

\begin{tabular}{|c|l|c|c|}
\hline No. & Formas & Totales & $\%$ Rel. \\
\hline 1. & Uso ejemplificativo & 582 & $47 \%$ \\
\hline 2. & Uso relativo de modo & 271 & $22 \%$ \\
\hline 3. & Uso comparativo de igualdad & 212 & $17 \%$ \\
\hline 4. & Uso relativo de lugar & 95 & $8 \%$ \\
\hline 5. & Uso del como conector causal & 47 & $4 \%$ \\
\hline 6. & Uso interrogativo indirecto & 23 & $2 \%$ \\
\hline 7. & Uso relativo de tiempo & 16 & $1 \%$ \\
\hline \multicolumn{2}{|c|}{ Total } & $\mathbf{1 2 4 6}$ & $\mathbf{1 0 0 \%}$ \\
\hline
\end{tabular}

A continuación, se ejemplifican los usos más frecuentes encontrados en CAELE:

\section{Uso Ejemplificativo}

Como se observa en los ejemplos (1) y (2), la característica de uso corresponde a la función que desempeña como atributo de los sintagmas nominales y el núcleo (sujeto). En el caso de la oración uno, el uso de como refiere a los atributos que se interpretan de los problemas de la sociedad, entre estos, problemas de salud mental. En el ejemplo (2), ocurre lo mismo, se identifica el uso de condición de atributo donde se ejemplifica el camino con atributos específicos con ramas y árboles. Este uso es muy característico de la ejemplificación, cuya función es determinada por el uso del como agregando y modificando atributos al sintagma nominal.

(1) "...Hay muchos problemas en la sociedad que afectan los jóvenes. Por ejemplo, la salud mental como depresión, la imagen físico, y violencia domestica" (Sujeto 54, nivel A2, L1 inglés). ${ }^{2}$

(2) "... Era un bus muy antiguo que no daba mucha confianza, ademas el camino tenía muchos ollos y obstaculos como ramas y arboles" (Sujeto 69, nivel B1, L1 francés).

\section{Uso relativo de modo}

En el ejemplo (3) se manifiesta su significado de relativo de modo equivalente a la expresión 'del modo que', es decir, "vivir del modo como viven los músicos". En el (4) se mantiene el mismo sentido en el uso del como, esto es, "La economía de Chile funciona del modo como la economía de los Estados Unidos." En este segundo ejemplo, el antecedente está explícito el sustantivo "economía". Como se puede ver en ambos ejemplos se expresa la función fundamental de introducir cláusulas que expresan una circunstancia modal de la oración principal (MARTíNEZ GARCÍA, 1989).

(3) "...Los músicos son el clave mapra mantener las tradiciones. Al orto lado parece como sea dificil vivir como músico" (Sujeto 9, nivel A2, L1 alemán).

(4) "...La economía de Chile funciona como la en los estados unidos entonces siempre bay esto problema abajo del superficie" (Sujeto 55, nivel B1, L1 inglés).

\section{Uso comparativo de igualdad}

Junto con las otras funciones propiamente relativas e interrogativas, como interviene en construcciones comparativas de igualdad con valores muy cercanos a los que tiene como adverbio relativo de modo. Las oraciones comparativas son oraciones degradadas, análogas a las relativas, con la diferencia de que su antecedente es un cuantificador o una unidad cuantificada. En el ejemplo (5), la estructura "disfruta tu viaje tanto..." contiene a tanto (cuantificador) y la estructura "...como disfruté..." contiene el conector comparativo (comparación cuantificada). En el ejemplo (6), el enunciado, cuyo verbo conjugado es contaminar, también se caracteriza por la presencia de un elemento cuantificador (más), seguido del comparativo (como).

(5) "Disfruta tu viaje a Chile tanto como disfruté lo mio" (Sujeto 53, nivel A2, L1 inglés).

(6) "...Las industrias chilenas contaminan más como las minas de cobres" (Sujeto 45, nivel B1, L1 francés).

2. Todos los ejemplos en este artículo están en itálico y corresponden a enunciados extraídos ipsis litteris 


\section{Uso relativo de lugar}

Respecto al relativo de lugar, se encontró un número interesante de ejemplos que ilustran un antecedente expreso de lugar correspondiendo también a las cláusulas relativas y en algunos otros casos sin antecedente expreso.

(7) "...Pero creo que como en Bolivia o en el Perú la artesanía es bonita" (Sujeto 18, nivel B1, L1 francés).

(8) "...Las ferias artesanales son más colorida como en el sur de Chile" (Sujeto 52, nivel B1, L1 alemán).

\section{Uso del como conector causal}

En cuanto al como causal, también se evidencia un uso menor respecto del total del corpus en estudio. No obstante, es un conector que para el nivel B1 debería tener mayor tendencia de uso. En estos resultados, se observa una aparición muy similar, la cual podría interpretarse como una estructura conocida o de fácil acceso para ambos niveles (A2-B1), puesto que el uso de esta palabra es común en diferentes oraciones.

(9) "...Como bay mucha gente que trabaja en esta ciudad, el trafico es borrible y causa una contaminación del aire muy importante..." (Sujeto 60, nivel A2, L1 francés).

(10) "...En Austria bay diferentes tipos de artesanía dependiente de la región. Como bay mucho bosque en Austria, bay muchos productos de madera..." (Sujeto 9, nivel B1, L1 alemán).

\section{Uso interrogativo indirecto}

El uso del como interrogativo en una oración interrogativa o exclamativa directa parcial puede ser transpuesta a la categoría del sustantivo con la pérdida de la curva melódica interrogativa y desempeñar funciones de complemento de implemento (yo sé cómo se baila) de suplemento (para decir cómo podemos solucionar) o de sujeto, aditamento y atributo del implemento (CUADRADO, 2002).

(11) "...Escucho casi todos los días reggeaton. Yo sé cómo se baila con este tipo de música. Para mí es divertido bailar en discotecas" (Sujeto 39, nivel A2, L1 alemán).

(12) "...Entonces, es claro que existe esas problemas en Chile y bay personas que están luchando contra las problemas. Es difícil para decir cómo podemos solucionar esas problemas porque son muy común y mucha gente creen en esas cosas malas" (Sujeto 56, nivel B1, L1 inglés).

\section{Uso relativo aproximativo de tiempo}

En este caso, se ha considerado como ejemplos un uso con valor aproximativo cuantitativo, es decir, se adjunta a circunstanciales para expresar aproximación o semejanza. En el ejemplo 13 se expresa un valor aproximativo relativo a la edad y en el ejemplo 14, la cláusula con la partícula como equivale a "(en los tiempos) durante la Guerra fría".

(13) "...Hoy los jóvenes empezan a tomar como a los 14 años..." (Sujeto 52, nivel A2, L1 francés)

(14) "... No tiene ningún apoyo como durante la Guerra Fría" (Sujeto 19, nivel A2, L1 francés).

Sobre la base de dichos resultados, se procedió a seleccionar los tres usos del como con mayor frecuencia para un análisis en mayor profundidad.

Tabla 3. Usos de la partícula como con mayor frecuencia

\begin{tabular}{|c|l|c|c|}
\hline No. & Formas & Total & $\%$ Rel \\
\hline 1 & Uso ejemplificativo & 582 & $55 \%$ \\
\hline 2 & Uso relativo de modo & 271 & $25 \%$ \\
\hline 3 & Uso comparativo & 212 & $20 \%$ \\
\hline \multicolumn{2}{|c|}{ Subtotal más frecuentes } & $\mathbf{1 0 6 5}$ & $\mathbf{1 0 0 \%}$ \\
\hline
\end{tabular}

Como se puede ver en la Tabla 3, la distribución de mayor a menor usos de la partícula como según sus frecuencias en el corpus de aprendientes de español como lengua extranjera corresponde a: (1) uso ejemplificativo con 582 
ocurrencias equivalentes al 55\% total de las tres estructuras más frecuentes encontradas en el corpus CAELE. Le sigue el uso relativo modal con 271 ocurrencias correspondientes al 25\% del total, y luego el comparativo de igualdad con 212 ocurrencias (20\%).

Estos resultados tienen relación con los indicadores especificados en los niveles de competencia A2 y B1 según el Plan Curricular del Instituto de Cervantes, en los que se especifica que la práctica y aprendizaje de los usos de la partícula comienza en el nivel principiante A2 e intermedio B1. También los resultados se pueden explicar en función de las estructuras textuales narrativas, descriptivas y argumentativas de los textos que integran el corpus CAELE.

\subsection{Los usos de como: atributo ejemplificativo, relativo de modo y comparativo}

En relación con el Objetivo 2 "delimitar los usos más frecuentes de acuerdo con el nivel de competencia y lengua materna de los aprendientes en estudio", en la Tabla 4 se presenta la distribución de los usos más frecuentes respecto al nivel de competencia A2 y B1 y las lenguas maternas de los aprendientes. Obsérvese la distribución por cada uso del adverbio como.

Tabla 4. Distribución de los usos de como por nivel de competencia

\begin{tabular}{|c|c|c|c|c|c|c|c|c|c|c|c|c|}
\hline Nivel y lengua & \multicolumn{4}{|c|}{ Nivel A2 } & $\%$ & \multicolumn{4}{|c|}{ Nivel B1 } & $\%$ & \multirow{2}{*}{ Totales } & $\%$ \\
\hline Formas & \begin{tabular}{|l|} 
Alemán \\
\end{tabular} & Francés & Inglés & Subtotal & Nivel A2 & Alemán & Francés & Inglés & Subtotal & Nivel B1 & & Total \\
\hline Uso ejemplificativo & 87 & 46 & 83 & 216 & $48 \%$ & 103 & 164 & 99 & 366 & $59 \%$ & 582 & $55 \%$ \\
\hline Uso relativo de modo & 39 & 43 & 45 & 127 & $28 \%$ & 40 & 69 & 35 & 144 & $23 \%$ & 271 & $25 \%$ \\
\hline Uso comparativo & 50 & 28 & 28 & 106 & $24 \%$ & 21 & 46 & 39 & 106 & $17 \%$ & 212 & $20 \%$ \\
\hline Subtotales Lengua & 176 & 117 & 156 & 449 & $100 \%$ & 164 & 279 & 173 & 616 & $100 \%$ & 1065 & $100 \%$ \\
\hline Totales por nivel & Total y $\%=$ & 449 & & $42 \%$ & & Total y $\%=$ & 616 & & $58 \%$ & & & \\
\hline
\end{tabular}

\section{a. Uso del como ejemplificativo}

En la Tabla 4 se visualizan las frecuencias de uso del como ejemplificativo según el nivel de competencia. Se observa en el nivel B1 la mayor frecuencia con un 59\% correspondiente a 366 usos con respecto a los otros dos usos en estudio el relativo modal y el comparativo. Sigue en cuanto a la segunda mayor frecuencia este mismo uso ejemplificativo con un 48\% en el nivel A2 correspondiente a 216 usos. De esta forma, se comprueba que, al contrastar los resultados de los usos en estudio, el uso de la partícula como ejemplificativo es el que mayormente utilizaron los aprendientes de la muestra ya sea francófonos, anglosajones o alemanes, con respecto a los otros usos de la partícula como.

En lo referido a la lengua materna en la Tabla 4 se puede observar que los aprendientes con L1 francés en el nivel B1 presentan el mayor número de usos en el atributo ejemplificativo con un total de 164 usos, muy por sobre las otras dos lenguas maternas y por sobre los otros dos usos en estudio, el relativo modal y el comparativo. Le siguen en dicho uso los aprendientes con L1 alemán de nivel B1 con 103 usos y anglosajones de nivel B1 con 99 usos. Se observa que en las tres lenguas el uso es mayor en el nivel de competencia B1.

Estas tendencias, más relevantes en la Tabla 4, reflejan que el uso del atributo ejemplificativo es el más usual, dada su funcionalidad similar en las lenguas maternas respecto de los atributos del sintagma nominal, que modifica al sujeto y cumple funciones atributivas. Obsérvese los siguientes ejemplos en las lenguas maternas de los aprendientes de ELE.

$\checkmark$ Des mesures concrètes, comme par exemple la libération de prisonniers politiques, seraient accueillies avec satisfaction (Francés).

$\checkmark$ Policies of segregation, direct and indirect, for example separate schools and housing areas (Inglés).

$\checkmark$ Öffentliche Projekte, wie zum Beispiel der Straßenbau, könnten kaum noch zeitnab umgesetzt werden (Alemán).

Algunos ejemplos del corpus CAELE corresponden a:

(15) "...Tiene varias origenes como indigenes o de otros continentes..." (Sujeto 36, nivel B1, L1 francés).

(16) "...Los artesanos bacen muchas cosas utiles como botas de cuerro, ollas de ceramica, monturas, arcos y flechas..." (Sujeto 17, nivel B1, L1 inglés).

(17) "...Se puede encontrar algunos temas, como el amor, la sociedad..." (Sujeto 29, nivel B1, L1 alemán). 


\section{b. Uso del como relativo de modo}

En la Tabla 4 se visualizan las frecuencias de uso de la partícula como en el uso de relativo de modo. Se puede ver que el nivel A2 presenta una mayor frecuencia en dicho uso, correspondiente a un $28 \%$ con respecto a los otros dos usos en estudio. En el nivel B1, en cambio el porcentaje en el relativo de modo disminuye en un $23 \%$ posicionándose como el segundo uso más frecuente del como en este nivel.

En cuanto a la lengua materna, en la Tabla 4 se muestra que el uso relativo de modo presenta una mayor frecuencia en los aprendientes de L1 francés en el nivel B1 con 69 usos, le sigue la frecuencia de uso de los aprendientes anglosajones de nivel A2 con 45 usos y los francófonos de nivel de competencia A2 con 43 usos.

Ejemplos de los resultados con mayor frecuencia.

(15) "Así que estoy aprendiendo como manejar los asuntos públicos..." (Sujeto 100, nivel B1, L1 francés).

(16) "como en otros países bay varias culturas y consideramos las culturas como la misma" (Sujeto 17, nivel A2, L1 inglés).

(17) "...también tiene el amor como un aspecto importante" (Sujeto, nivel B1, L1 alemán).

Se puede verificar el mayor dominio de esta estructura de relativo modal en los aprendientes francófonos en ambos niveles de competencia, esto puede deberse a la similitud de las cláusulas relativas de modo en ambas lenguas.

\section{c. Uso del como comparativo}

En la Tabla 4 se puede delimitar que el nivel de competencia A2 presenta una mayor frecuencia en el uso del comparativo de igualdad con un 24\% en relación con el nivel B1 que presenta un 17\%. Estos resultados se encuentran en consonancia con los contenidos gramaticales expuestos en el Plan Curricular, pues es en el estadio de aprendizaje A2, en el cual los aprendientes comienzan a producir estructuras comparativas. El mismo Plan Curricular menciona que nivel B1 se caracteriza por la producción de oraciones compuestas por cláusulas subordinadas, caracterizadas por una mayor complejidad sintáctica (INSTITUTO CERVANTES, 2006).

Con respecto a la lengua materna se puede determinar que en los aprendientes alemanes en el nivel de competencia A2 se observa la mayor frecuencia de uso del comparativo con 50 usos, seguido por los aprendientes francófonos de nivel B1 con 46 enunciados y por los anglosajones con 39 usos del como en estructura comparativa.

Ejemplos:

(19) "... La posición de la familía no es tan importante como en otras país del sur de America y el imadén de la familía no es muy tradiciona" (Sujeto 62, nivel A2, lengua alemán).

(20) "... En conclusión, creo que para comer carne no es tan malo si comes carne en moderación y si la carne que comes es de un fuente mejor como una granja orgánico de su región" (Sujeto 54, nivel A2, lengua inglés).

(21) "Creo que los chilenos no valoran suficiente el artesanía porque es una cosa muy importante, es como el alma del país" (Sujeto 6, nivel B1, lengua francés).

En la Tabla 5 se muestran los usos totales de la partícula como de acuerdo con las tres lenguas en estudio. La tabla contiene la frecuencia absoluta de cada uso y su frecuencia relativa respecto del total por lengua. En la columna final, se exponen los porcentajes respecto del total de usos de las tres lenguas, esto es 1065 usos en total.

Tabla 5. Tendencias de uso del como según la lengua materna

\begin{tabular}{|l|c|c|c|c|c|c|c|c|}
\hline Formas & Alemán & $\%$ Rel. & Inglés & $\%$ Rel. & Francés & $\%$ Rel. & Totales & $\%$ Rel. \\
\hline Uso ejemplificativo & 190 & $18 \%$ & 182 & $17 \%$ & 210 & $20 \%$ & 582 & $55 \%$ \\
\hline Uso relativo de modo & 79 & $7 \%$ & 80 & $8 \%$ & 112 & $11 \%$ & 271 & $25 \%$ \\
\hline Uso comparativo & 71 & $7 \%$ & 67 & $6 \%$ & 74 & $7 \%$ & 212 & $20 \%$ \\
\hline Subtotales Lengua & 340 & $32 \%$ & 329 & $31 \%$ & 396 & $37 \%$ & $\mathbf{1 0 6 5}$ & $\mathbf{1 0 0 \%}$ \\
\hline
\end{tabular}

Como se puede constatar en la Tabla 5, el uso del como en sus tres formas revela una consistencia entre las lenguas de estudio. Es decir, la diferencia del uso por cada lengua oscila entre 20 y 50 usos registrados por lengua. Esto podría indicar que la funcionalidad del como en sus tres formas tendría similitudes de acuerdo con la lengua 
materna. De alguna manera, su funcionalidad en el sistema lingüístico parece transferirse de acuerdo con el uso del español.

En relación con los usos por cada lengua, por un lado, se observa, por ejemplo, que en el alemán e inglés el uso del atributo ejemplificativo tiene una frecuencia cercana del $18 \%$ de uso a diferencia del francés con 2 puntos de porcentaje mayor, 20\%. Este uso representa el 55\% de toda la muestra. Por otro lado, en el uso del relativo de modo, ocurre algo similar, observándose una diferencia de sólo un punto de frecuencia, correspondiente al 7\% en alemán y el $8 \%$ en inglés. En cambio, en francés, la diferencia radica en 3 puntos más, con una representación del 11\%. En total, el uso de relativo de modo representa el $25 \%$ del total de usos.

Finalmente, en relación con el uso comparativo, se observa la misma tendencia, pero a diferencia de los otros dos usos, el francés mantuvo la similitud con las demás lenguas. Por ejemplo, en el alemán se expone un 7\% de uso, similar al caso del inglés con un $6 \%$ y, el francés, con un $7 \%$. Esto podría interpretarse como una forma de uso equiparable o similar en las tres lenguas. Para tener una mayor diferenciación de los usos, se presenta el siguiente gráfico con los usos por frecuencia absoluta.

El Gráfico 2 muestra la distribución de los usos de como según la variable lengua. Los aprendientes francófonos produjeron 74 enunciados con el uso del como comparativo; seguido de los aprendientes del alemán que produjeron 71 usos; finalmente, los aprendientes anglosajones produjeron 67 usos comparativos, en total 212 usos. En el francés, la supremacía de usos de la partícula como se podría deber a la similitud del sistema lingüístico con el español, por lo que podría configurarse como una variable que estaría incidiendo en una producción caracterizada por combinaciones con la partícula como.

Gráfico 2. Total de frecuencias de usos por lengua materna

\section{FRECUENCIA POR LENGUA}

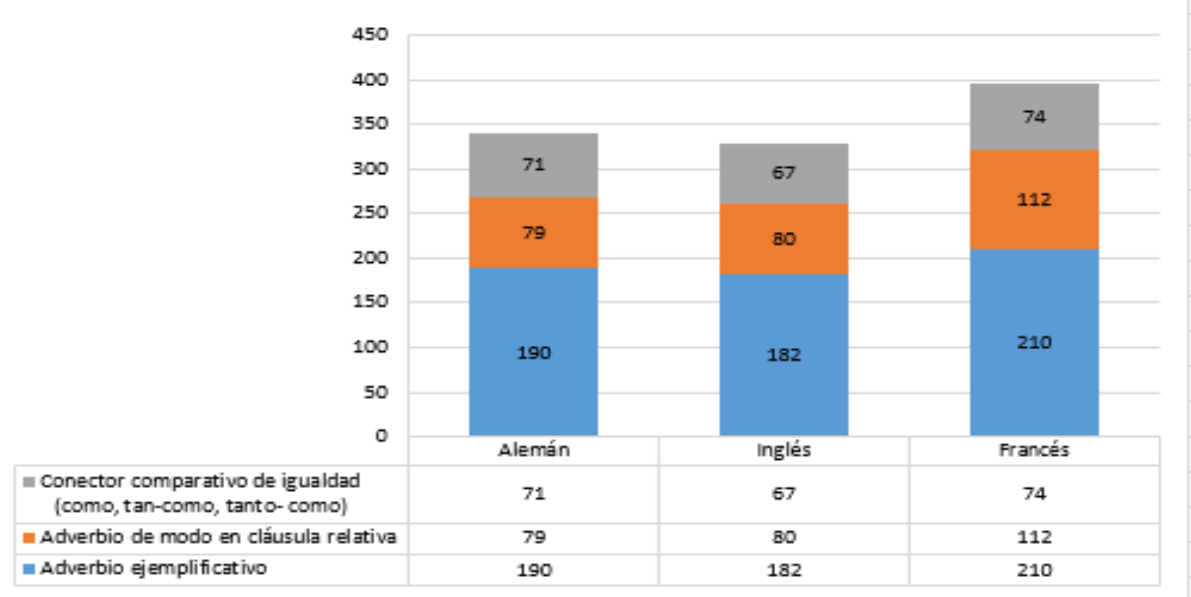

En general, los resultados muestran un mayor uso de la partícula como en el caso de los aprendientes francófonos en ambos niveles de competencia. Como se trata de dos lenguas romances hay mayor cercanía entre las formas de uso y funcionalidad del como y comme en ambas lenguas. Esto se ilustra en el siguiente listado de usos del comme en francés.

1. Uso comparativo: mes amis comme les tiens sont tous venus tanto mis amigos como los tuyos han venido. il écrit comme il parle (escribe como habla).

2. Uso para indicar manera: comme ça, tu seras content (así estarás contento).

3. Uso para indicar atributo ejemplificativo les mammifères comme par exemple le dauphin (los mamíferos como por ejemplo el delfín).

Finalmente, en lo que corresponde a las frecuencias de uso en los niveles de competencia A2 y B1 en las estructuras en estudio, el Gráfico 1 muestra la diferencia de los 3 usos más frecuentes de la partícula como. La distribución es similar en ambos niveles en cuanto a los totales de los usos más frecuentes, es decir, tanto en el nivel A2 (20\%) como en el nivel B1 (34\%), el adverbio con valor ejemplificativo es el más usado por los aprendientes de $\mathrm{ELE}_{;}$en el nivel intermedio B1 el uso es notoriamente mayor (34\%). La presencia del como en cuanto a adverbio de modo en cláusulas relativas es el segundo uso más utilizado por los aprendientes del corpus digital y la diferencia 
entre el nivel A2 (12\%) y B1 (14\%) es muy cercana. Al igual que en la partícula anterior, el nivel intermedio B1 se caracteriza por mayor cantidad de usos (14\%). Finalmente, ambos niveles se caracterizan por el mismo porcentaje (10\%) en la producción de oraciones comparativas de igualdad (10\% cada uno).

Gráfico 1. Total de frecuencias de uso por nivel de competencia.

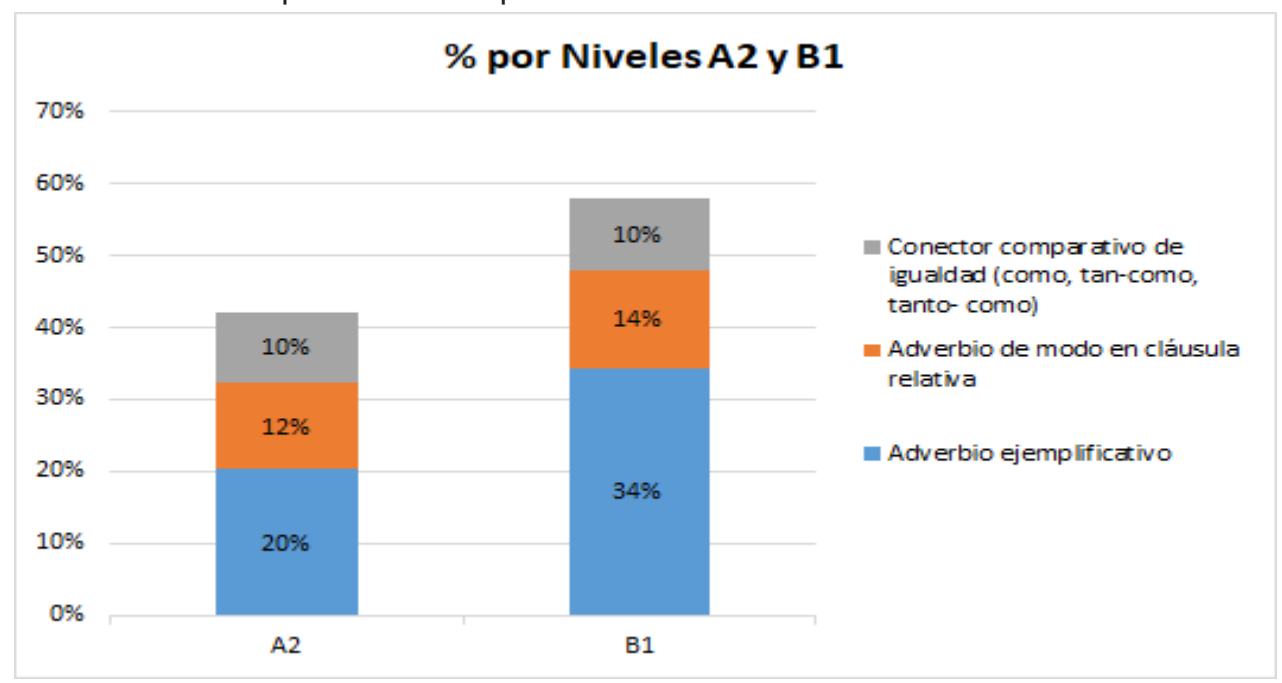

\section{CONCLUSIONES}

En este artículo, hemos presentado un estudio descriptivo con un enfoque de análisis de datos mixto con el propósito de explorar un corpus de aprendientes de Español como Lengua Extranjera, CAELE, las tendencias de uso correcto e incorrecto que se observan en aprendientes anglosajones, alemanes y francófonos de nivel A2 y B1 con respecto al usos de la partícula en un contexto de tareas de escritura académica.

En el marco de esta investigación, el estudio realizado a partir del CAELE respecto de las tendencias de uso de la partícula como aporta información relevante sobre los procesos que sigue el aprendiente de acuerdo con la propuesta de enseñanza del Plan Curricular del Instituto de Cervantes y el logro del aprendizaje en cada temática que aborda por nivel. Los resultados de la investigación muestran que las tendencias de uso del como ejemplificativo, relativo modal y comparativo en el corpus digital CAELE, en general, se condicen con el nivel de competencia de los estudiantes. De esta manera, los datos permiten identificar y determinar los usos de la partícula como que presentan dificultades para el aprendizaje de los estudiantes de acuerdo con su lengua materna y el nivel de competencia lingüística.

Los resultados revelan que el uso de la forma como en relación con las distintas funciones que desempeña, puede presentarse en funciones que registran desde la ilustración o condición de atributos, hasta las que coinciden como conectores, adverbio interrogativo y relativos tanto de tiempo como de lugar. En esta línea, respecto de las tendencias de uso más frecuentes del CAELE, se observa una mayor aparición de tres formas en funciones específicas de la partícula como. La primera referida al uso ejemplificativo, la segunda en su función de modo relativo y, por último, el uso comparativo. Estas tres formas se condicen con el uso frecuente en las situaciones comunicativas tanto en español como lengua materna como en el español como lengua extranjera, ya que el discurso escrito u oral se apoya de distintos recursos para complementar o reforzar las ideas centrales. Por lo general, en el discurso escrito hay una tendencia marcada hacia la ejemplificación como forma de reforzar las explicaciones o definiciones que a diario realizamos en distintas temáticas. Del mismo modo, ocurre con el uso relativo de modal, dado que en muchas situaciones comunicativas se expresa la forma en la que ocurren las cosas o el modo como se conciben las situaciones u otras formas de representación de la cotidianeidad. En el caso de las comparaciones, es otro uso frecuente, puesto que, para complementar o entregar mayor fuerza en el discurso, siempre se recurre no sólo a la ejemplificación, sino también al contraste entre las diferentes opciones que aparecen, plasmándose así un contraste o comparación que complementa la definición o explicación dada con anterioridad.

En el caso del uso de atributo ejemplificativo, esta tendencia puede explicarse en términos del uso del como de forma que cumple la función de condición de atributo, cuyo fin es ilustrar con mayor detalle una oración antecedente 
o sujeto antecedente de forma cuantificada atribuible a una o varias características. Este uso es común tanto en la lengua materna como extranjera, ya que es una secuencia oracional que responde a diferentes formas de expresar la información en un texto, ya sea expositivo, informativo o descriptivo. No obstante, es escasa la bibliografía respecto de este uso, ya que puede asociarse en muchos casos como un conector de ejemplificación. Sin embargo, puede determinarse claramente que cumple una función específica de atributos del sujeto o núcleo del sintagma nominal.

Para finalizar, estas tendencias de uso son relevantes y fortalecen la aún escasa literatura existente sobre la partícula como en el ámbito de Español como Lengua Extranjera. En consecuencia y dentro del campo de estudio de las implicaciones didácticas, proponemos: a) explicitar y explicar los usos más representativos de esta partícula en las clases de ELE, debido a que es una forma compleja y que incluso puede generar dificultad en las producciones escritas de español como lengua materna y b) crear y/o utilizar materiales auténticos considerando diversos contextos que favorezcan el proceso de enseñanza y aprendizaje del como. Los hallazgos de este estudio aportan al área de la Lingüística Aplicada, adquisición y enseñanza del Español como Lengua Extranjera, contribuyendo con resultados reales de tendencias de uso de la partícula como, sugiriendo incorporar en las propuestas de enseñanza una mayor práctica y explicación de las estructuras lingüísticas implicadas en cada uno de ellos. Asimismo, en la formación de los profesores de ELE se debe orientar en la selección y disposición de los materiales y tareas auténticas que respondan a la realidad lingüística y sociocultural de los aprendientes del español y que consoliden el aprendizaje, la enseñanza y la evaluación de la lengua meta.

\section{REFERENCIAS}

ACÍN VILLA, E (2001). "Algo más sobre como". Anuario de Estudios Filológicos, XXIV, pp. 9-24.

BELLO, A. (1988). Gramática de la lengua castellana destinada al uso de los americanos. Madrid: Arco Libro.

BRUCART, J. M. (1999). "La estructura del sintagma nominal: las oraciones de relativo", en Bosque y Demonte (dirs.), tomo 1, pp. 395-522.

CANO AGUILAR, R. (1995). Sintaxis bistórica de la comparación en español. La bistoria de como. Sevilla: Universidad de Sevilla.

CONSEJO DE EUROPA. (2001). Marco Común Europeo de Referencia para la Enseñanza de las lenguas. Madrid: Instituto Cervantes-MECD, Anaya.

CUADRADO, L. (2002). Sobre el funcionamiento de como en español. Revista de Filología Románica, 19, pp. 325-340.

DÍAZ RODRÍGUEZ, L.; MARTÍNEZ SÁNCHEZ, R.; REDÓ BANZO, J. (1964). Guía rápida del "Marco" para ELE: contenidos linguísticos por niveles para el Marco Europeo de referencia para la enseñanza de las lenguas. Barcelona, ReGAE.

FERREIRA, A. (2016). "Prueba de multinivel con fines específicos académicos". Universidad de Concepción, Chile.

FERREIRA, A. (2018-2021). FONDECYT- CONICYT N 1180974. "Diseño e implementación de un corpus escrito de aprendientes de ELE en formato computacional para el análisis de la interlengua". Proyecto de Investigación. CONICYT, Chile.

FERREIRA, A.; ELEJALDE, J. (2019). Hacia un perfil lingüístico-comunicativo del estudiante de Español como Lengua Extranjera para fines Académicos. Revista Nebrija De Lingüística Aplicada a La Enseñanza De Lenguas. v.13, n. 27, pp145-165.

FERREIRA, A.; BLANCO, L.; ELEJALDE, J. (2020). "Análisis de Errores en el Corpus CAELE: estudio sobre la concordancia gramatical en el verbo SER en aprendientes francófonos y anglosajones". Revista Nebrija De Lingüística Aplicada a La Enseñanza De Lenguas. v. 14, n.29, p.76-99.

FERREIRA, A.; ELEJALDE, J. (2020). Propuesta de una taxonomía etiológica para etiquetar errores de interlengua en el contexto de un corpus escrito de aprendientes de ele". Forma y Función. v. 33, n.1, p. 115-146.

GONZÁLEZ GARCÍA, L. (1995). "Así y como, fóricos textuales", Moenia 1, pp. 315-329. 
GRANGER, S. (2015). Contrastive interlanguage analysis: A reappraisal, International Journal of Learner Corpus Research. v.1, n1, p.7-24.

HASSELGARD, H.; JOHANSSON, S. (2011). "Learner corpora and contrastive interlanguage análisis". In: F. Meunier, S. De Cock, G. Gilquin y M. Paquot, (eds), A taste for corpora: In bonour to Sylviane Granger, $1^{\text {a }}$ ed, Amsterdam/Philadelphia: John Benjamins Publishing Company, p. 33-62.

INSTITUTO CERVANTES. (2006). Plan curricular del Instituto Cervantes. Niveles de Referencia para el español, Madrid: Instituto Cervantes- Biblioteca Nueva.

JIMÉNEZ, J. (2003). "Como en español actual". Verba. v 30, n.1, p.117-161.

KILGARRIFF, A.; RYCHLÝ, P.; SMRŽ, P.; TUGWELL, D. (2004). The Sketch Engine. Information Technology. pp.105, 116.

KILGARRIFF, A.; BAISA, V.; BUŠTA, J.; JAKUBÍČEK, M.; KOVÁŘ, V.; MICHELFEIT, J.; RYCHLÝ, P.; SUCHOMEL, V. (2014). The Sketch Engine: Ten years on. Lexicography, 1, pp. 7-36.

MARTÍNEZ GARCÍA, J.A. (1989). El pronombre. II. Numerales, indefinidos y relativos. Madrid: Arco Libros.

MORERA, M. (1990). "El adverbio relativo como: significado y usos", en Actas del Congreso de la Sociedad Española de Lingüística, XX Aniversario, tomo II, pp. 681-691.

REAL ACADEMIA ESPAÑOLA. (2005). Diccionario Panbispánico de dudas, $1^{\text {a }}$ ed. Madrid: Taurus.

REAL ACADEMIA ESPAÑOLA. (2010). Manual de la Nueva Gramática de la Lengua Española. Buenos Aires: Espasa.

REAL ACADEMIA ESPAÑOLA. (2014). Diccionario de la lengua española, 23 a ed.. Buenos Aires: Espasa.

SÁNCHEZ, A. (2015). La Investigación de Corpus de Aprendientes y el desarrollo de los estudios de la interlengua del español, Language design: Journal of theoretical and experimental linguistics. v.17, n.1, p. 57-83.

TRUJILLO, R. (1990). "Sobre la explicación de algunas construcciones de como", Verba, 17, pp. 249-266.

Recebido: 8/6/2021

Aceito: 20/10/2021

Publicado: 27/10/2021 